\section{Beugen oder Strecken? Ellenbogen-Winkel beeinflusst Blutdruckmessung}

\begin{abstract}
W er den Blutdruck mal am gestreckten und mal am gebeugten Arm misst, darf sich über ein Auf und $\mathrm{Ab}$ der Werte nicht wundern. Zwar sind die Schwankungen nicht gigantisch, aber bei einer exakten Blutdruckeinstellung durchaus von Bedeutung. Fehlerquellen gibt es bei der Blutdruckmessung genügend: Falsche Manschetten-Größe, volle Harnblase, Reden während der Messung oder Weißkitteleffekt können schnell zu unbrauchbaren Werten führen. Als weiteres Problem haben amerikanische Kollegen von der University of California nun die Haltung des Unterarmes entdeckt.

In einer prospektiven Studie fanden sie bei insgesamt 100 Patienten signifikant niedrigere Werte, wenn der Arm der Probanden im Ellenbogen-Gelenk nicht gestreckt, sondern $90^{\circ}$ gebeugt war. Untersucht wurde dieser Effekt jeweils am liegenden, sitzenden und stehenden Patienten, wobei die Manschette auf Herzhöhe angebracht war. Die größten Unterschiede fanden sich bei den LiegendMessungen, die am gestreckten Arm eine durchschnittliche Erhöhung des systolischen Blutdruckwertes um 13,2 mmHg ergaben und für den diastolischen Wert um 14,4 mmHg. Im
\end{abstract}

Sitzen und Stehen fielen die Differenzen zwar etwas geringer aus, waren aber ebenfalls signifikant, wie die Untersucher in der aktuellen Ausgabe der Annals of Internal Medicine berichten.

Die Unterschiede erachten die Autoren für die Praxis durchaus als relevant, da die Abweichungen den Bereich von 5 bis $10 \mathrm{mmHg}$ übersteigen, der nach ihren Erläuterungen bei der Blutdruckeinstellung von Bedeutung ist. Dabei verweisen sie auf ihre Studien-Ergebnisse, wonach 41\% der Patienten bei den SitzendMessungen mit gestrecktem Arm in die Klassifikation Bluthochdruck gefallen wären. Dagegen bei gebeugtem Arm nur 22\%. Ob der Blutdruck nun korrekterweise bei gestrecktem oder gebeugtem Arm zu messen ist, lassen die amerikanischen Kollegen allerdings offen. Zukünftige Blutdruckstudien sollten nach ihrer Meinung der Arm-Haltung jedoch mehr Bedeutung beimessen. Zudem sollte man in der täglichen Praxis darauf achten, dass man auch tatsächlich immer die gleiche Arm-Position wählt. Karl Eberius, Heidelberg

Quelle: Thomas J. Hemingway et al. Annals of Internal Medicine 2004; 140: 74

Tab. 1 Fallstricke bei der Blutdruckmessung

\begin{tabular}{|lll} 
& $\begin{array}{l}\text { Einfluss auf syst./diast. } \\
\text { Wert }(\mathbf{m m H g})\end{array}$ & Evidenz-Klasse \\
\hline Manschette über der Kleidung & bis zu $+50 /+50$ & IV \\
\hline Dehnung von Harnblase oder Darm & $+27 /+22$ & IV \\
\hline Unterhalten & $+17 /+13$ & I \\
\hline Akute Kälteexposition & $+11 /+8$ & II \\
\hline Manschette zu schmal & $-8 /+8$ & I \\
\hline Alkoholkonsum & $+8 /+7$ für drei Sunden & \\
& oder weniger & I \\
\hline Armhaltung & $\pm 8 / \pm 8$ pro 10 cm über oder & I \\
& unter der Herzhöhe & IV \\
\hline Erwartungsbias & Rundung auf die nächsten & \\
\hline Körperliche Aktivität & $5-10 / 5-10$ & \\
\hline Die Beispiele sind www.evidence.de entnommen & -5 bis $-11 /-4$ bis -8 für & \\
\hline
\end{tabular}

\section{Screening-Verfahren Fahrtüchtigkeit bei Demenz}

eichte Demenz gilt als das erste Stadium für den Verfall der geistigen Fähigkeiten bei Alzheimer-Patienten. Amerikanische Wissenschaftler haben ein Screening-Verfahren entwickelt, das festlegt, ab welchem Zeitpunkt Menschen mit leichter Demenz nicht mehr fahrtüchtig sind. Demnach liefern visuell-räumliche Tests die besten Vorhersagen zu den jeweiligen Fahrfähigkeiten. Diese Tests, die Situationen auf und jenseits der Straßen simulierten, waren hinsichtlich der neuropsychologischen Funktionen den Berichten von Pflegenden überlegen. Es zeigte sich, dass das Testen von Aufmerksamkeit und Konzentration weniger aussagekräftig war.

\section{Visuell-räumliche Tests liefern die besten Ergebnisse}

Analysiert wurden 27 Studien, die zwischen 1988 und 2003 die Beziehung zwischen neuropsychologischen Funktionen und der Fahrtüchtigkeit von Demenz-Patienten untersuchten. Obwohl Defizite im visuell-räumlichen Bereich alleine noch nicht ausreichen, um Einschränkungen bei der Teilnahme am Straßenverkehr zu empfehlen, macht das Vorhandensein dieser Defizite eine sorgfältige Untersuchung weiterer Risikofaktoren sinnvoll.

\section{Erhöhtes Unfallrisiko}

Die Ergebnisse zeigten, dass Demenz-Patienten einem erhöhten Unfallrisiko ausgesetzt sind und eher die Orientierung verlieren. Der progressive Verlauf einer Demenz verkompliziert Screening-Verfahren für jene, die unter leichten Symptomen leiden. Neuropsychologische Tests können einen entscheidenden Beitrag zur Einschätzung der Fahrtüchtigkeit liefern. Die Ergebnisse der Studie wurden im Fachmagazin Neuropsychology veröffentlicht (http://www. apa.org/journals/neu. html).

Quelle: pressetext.austria, pte.online 